\title{
The relationship between the airborne fungi and allergic childhood asthma in Wuhan
}

\author{
Dong Zong-Qi \\ (Wuhan Children's Hospital)
}

Objective : To study the relationship between the airborne fungi and allergic asthma in children.

Methods: Airborne fungi were isolated and identified by using gravitational precipitation method (sheet glass and culture plate of exposure) determination. Fungal allergen skin tests were performed on 345 children with bronchial asthma. Fungal immunotherapy was given to children who showed positive reaction on the skin tests.

Results : Observation of 35,746 grain of airborne fungi spores and 1,188 grain of fungi spores for one year revealed the follwing species of fungi:Ustilaginales, Aternaria uredinales, Cladosporium cladosporioides, Cladosporium pithomyces, Cladosporium macrocarpum, Aspergillus niger, Aspergillus versicolor and Aspergillus fumigatus. Thirty-nine $(45.9 \%)$ of $85(41.5 \%)$ of 260 older children with asthma were positive for the skin tests; total positive rate was $42.6 \%$. Eighty-one in 108 cases of asthma completed fungal immunotherapy. Total effective rate was $88.0 \%$.

Conclusion: The airborne fungi are important sensitization allergen for inducing asthma in children. Fungal immunotherapy in an effective therapeutic method of asthma associated with fungi. 